\title{
Immunohistochemical Analysis of Expression of GATA3 in Carcinoma Breast and its Correlation with Prognostic Parameters
}

\author{
Tanvi Suri* and Charanjeet Ahluwalia
}

Department of Pathology, Vardhman Mahavir Medical College and Safdarjung Hospital, New Delhi, 110029

\begin{abstract}
Background: GATA3 plays an essential role in the normal development and function of the mammary gland where it promotes the luminal transcriptional program. Its loss is implicated in the pathogenesis of breast cancer. We proposed to study the expression of GATA3 in carcinoma breast by immunohistochemistry and determine its correlation with prognostic parameters.

Methods: The expression pattern of GATA3 was evaluated by immunohistochemistry in 30 cases of invasive breast carcinoma. GATA3 scoring was done and a score of $\geq 1+$ was considered positive. Patient characteristics, including age, tumour laterality, tumour size, lymph node status, tumour grade, histological type, molecular subtypes were collected. The relationships between protein expression and clinicopathological variables were analysed. Statistical significance was determined by Pearson's chi-square test and Mann Whitney U test (for age).

Result: $46.7 \%$ of cases (14/30) scored positive for GATA3 expression in tumour cells including $63 \%$ of luminal subtypes, $14 \%$ of Her-2 neu enriched carcinomas and $20 \%$ of triple negative carcinomas. Most positive cases $(35.7 \%)$ demonstrated $3+$ staining. GATA3 expression showed an inverse association with histological grade $(\mathrm{P}=0.012)$ and HER-2 overexpression $(\mathrm{P}=0.038)$, and a direct association with ER expression ( $\mathrm{P}=0.017)$ and $\mathrm{PR}$ expression $(\mathrm{P}=0.009)$.

Conclusion: GATA3 is luminal marker as it shows strong association with ER and PR in breast cancers. High GATA3 expression is also correlated good prognostic parameters like low tumour grade. Our findings advocate for GATA3 as a promising new breast-specific immunomarker.
\end{abstract}

Keywords: GATA3, Breast Carcinoma, Immunohistochemistry, Tumor Marker, Surrogate Molecular Classification

\section{Introduction}

Breast cancer is the most commonly diagnosed cancer and the leading cause of cancer death in females, worldwide with $20,88,849$ new cases $(11.6 \%)$ and $6,26,679$ deaths (6.6\%). 2018 saw 1,62,468 new cases (15.46\%) and 87,090 deaths $(12.11 \%)$ due to breast cancer in India. ${ }^{[1]}$ With age adjusted rate as high as 25.8 per 100,000 women and mortality 12.7 per 100,000 women, breast cancer is a major cause of morbidity and mortality especially in the urban Indian population. ${ }^{[2]}$

GATA binding protein 3 (GATA3) is one of six members of a family of zinc finger transcription factors which was first identified as a regulator of immune cell function. It plays a crucial regulatory role in the development of hematopoietic cells, skin, breast, kidney and central nervous system. GATA3 is responsible for maintaining the quiescent state of differentiated luminal cells in the adult mammary gland, therefore it is probably causally involved in pathogenesis of carcinoma breast. ${ }^{[3]}$ Supporting this hypothesis, a study done on a mouse model revealed that loss of GATA3 is associated with loss of tumour differentiation, marked progression from adenoma to early carcinoma and onset of tumour dissemination whereas restoration of GATA3 in late carcinomas brought about tumour differentiation and suppressed tumour dissemination. ${ }^{[4]}$ Although GATA3 is expressed in a wide variety of tissues, GATA3 labelling has been shown to be a highly specific marker for breast carcinomas and urothelial carcinomas only ${ }^{[5]}$

A number of previous studies have demonstrated high GATA3 levels in ER-positive (luminal) breast cancers. They found that GATA3 and estrogen receptor (ER) are involved in a cross-regulatory loop and are therefore frequently co-expressed in breast cancers. ${ }^{[6-14,20]}$ But expression of GATA3 in triple-negative (ER, PR, and HER2 negative) breast carcinomas is most relevant because this molecular subtype exhibits a negative immunohistochemical profile for most markers of breast origin. Due to low sensitivities of mammaglobin and GCDFP15, GATA3 may potentially be a useful marker in triple negative breast cancer ${ }^{[15,16]}$

The association between GATA3 and the prognosis of various cancers have been a research topic of considerable interest. Several studies have been conducted to evaluate expression of GATA3 in breast cancer. However, there is paucity of literature for such studies in Indian patients. Moreover, the results obtained from these studies have not been uniform and the clinical use of immunohistochemistry 
of GATA3 remains questionable. Some studies demonstrate it to be an independent prognostic marker ${ }^{[7-10]}$ while others show it lacks prognostic benefit. ${ }^{[12,18,14]}$

Determination of prognosis and identification of suitable treatment modalities remain the most immediate challenges after diagnosis of carcinoma breast. Markers to evaluate risk of relapse and metastasis are largely lacking. Since GATA3 represents a potential prognostic biomarker in carcinoma breast especially in the luminal subtype, its expression needs to be assessed.

\section{Objectives}

1) To perform immunohistochemistry using anti-GATA3 monoclonal antibody on samples of carcinoma breast and to score GATA3 immunostaining semiquantitatively.

2) To correlate GATA3 immunohistochemistry with prognostic parameters-

a. histological grade (modified Bloom-RichardsonElston grading system)

b. surrogate molecular classification

c. pTNM (wherever possible)

\section{Materials and Methods}

Study design and duration: This hospital-based crosssectional study was conducted over a period of 2 months (July-August 2019) in the Department of Pathology, Safdarjung Hospital after obtaining clearance from the Institute Ethics Committee. Informed written consent was taken from each patient.

Sample size and study population: A minimum of 30 samples satisfying the inclusion and exclusion criteria were included in this study.

Inclusion criteria: All histopathologically proven cases of invasive carcinoma breast in female patients.

Exclusion criteria: a. Patients on chemotherapy/ radiotherapy. b. Sarcomas, metastatic lesions, benign lesions and in situ carcinomas of breast.

Clinical Details: Routine clinical details like MRD No., Name, Age, Sex, Clinical Diagnosis were taken for every patient.

\section{Collection \& preparation of material:}

Procedure: Core needle biopsies of breast tissue \& mastectomy specimens were obtained in $10 \%$ formalin and representative tissue were grossed and processed as routine. Routine 4 to $5 \mu \mathrm{m}$ sections were cut and sections were stained with hematoxylin and eosin as per the standardized procedure. $^{[21]}$
Every stained section was evaluated for the following: -

a. Histological typing of the tumor

b. Histological grading of the tumor, according to the modified Bloom-Richardson-Elston grading system as Grade 1 (well differentiated), Grade 2(moderately differentiated), Grade 3 (poorly differentiated). ${ }^{[22]}$

c. Pathological Staging pTNM was assessed, wherever possible. ${ }^{[23]}$

Immunohistochemistry: Immunohistochemistry (IHC) for Estrogen Receptor (ER), Progesterone Receptor (PR), Human epidermal growth factor receptor 2 (HER2/neu), Ki 67 and GATA3 was performed using their respective antibodies on formalin fixed, paraffin embedded breast tissue specimens. GATA3 IHC was done using prediluted mouse monoclonal antibody. (Biocare Medical). Immunohistochemistry for ER, PR, HER2/neu and Ki67 was used to assess Surrogate Molecular Classification which is as follows ${ }^{[22]}$ :-

a. Luminal A- ER, PR positive and Ki $67<14 \%$

b. Luminal B- ER, PR positive; HER2/neu negative/ positive and $\mathrm{Ki} 67>/=14 \%$

c. Basal type or Triple Negative- ER, PR, HER2/neu negative

d. HER2/neu Enriched- HER2/neu positive

GATA3 score: Scoring of GATA3 immunostaining is semiquantitative. GATA3 labelling was scored on a scale of 0 to $4+$ with the extent of nuclear staining graded as follows ${ }^{[5]}: 0(0-5 \%), 1+(6 \%-25 \%), 2+(26 \%-50 \%), 3+$ $(51 \%-75 \%), 4+(>75 \%)$

For statistical analysis, we dichotomized GATA3 staining into negative ( 0$)$ and positive (1+ or more). Intensity of staining was recorded separately as weak, moderate, or strong. Any intensity of staining with greater than $5 \%$ distribution was considered positive. Ductal epithelial cells in benign lobules are the positive internal controls for GATA3 staining. ${ }^{[12,15]}$

Statistical Analysis: The data was entered in Microsoft Excel Spreadsheet. The data was analyzed using licensed SPSS software version 21.0. The data has been presented in tables and appropriate diagrams. Quantitative data has been summarized as mean and standard deviation while qualitative data has been summarized as proportions. The association between different groups for qualitative variable was analyzed by Pearson's chi square test/Linear by linear association test and for quantitative variable by Mann Whitney U test. A p value $\leq 0.05$ will be considered statistically significant. 


\section{Result}

Patient and Clinicopathological Data: Thirty female cases of invasive breast carcinomas were included in the study. The age at diagnosis of the patients ranged from 24 to 84 years, with a median age of 50 years. Mean age was $51.3 \pm 11.38$ years. $40 \%$ of patients were older than 51 years. Similar to the general patient population, most of the cases were infiltrating ductal carcinoma (96.6\%). This cohort of primary invasive ductal carcinomas consisted of 9 luminal A carcinomas (30\%), 10 luminal B carcinomas (33.4\%), 6 HER2/neu enriched carcinomas (20\%), and 5 triple negative carcinomas $(16.6 \%)$. The important clinicopathological data is presented in Table 2.

GATA3 Immunostaining: Immunoexpression of GATA3 was observed in the nuclei of malignant cells, as well as in some benign luminal epithelial cells from adjacent normal ducts (serving as a positive internal control).

14 of the 30 cases (46.7\%) scored positive for GATA3 expression in tumour cells including $63.2 \%$ of luminal subtypes, $16.7 \%$ of HER2/neu enriched carcinomas and $20 \%$ of triple negative carcinomas. Most positive cases $(35.7 \%)$ showed $3+$ staining. The intensity of staining was found to range from moderate to strong in the luminal $\mathrm{A}$ and luminal B subgroups, and weak to moderate/strong in the Her-2 and triple-negative subtypes. The distribution of GATA3 scores is displayed in Table 1.

Association with Clinicopathological Characteristics: GATA3 expression showed a significant correlation with luminal subtype of breast cancer (Pearson $\chi^{2}$ test, $P=0.017$ ) as $63.2 \%$ of luminal breast cancers were GATA3 positive versus only $18.2 \%$ of the non-luminal subtype (Figure 1 ).

Of the ER positive cases (60\% of the patient cohort), $66.7 \%$ were GATA3 positive. On the other hand, among the ER negative cases, only $16.7 \%$ were GATA3 positive. A strong association between GATA3 protein expression and positive ER status was observed (Pearson $\chi^{2}$ test, $P=0.007$ ). Altogether, $85.7 \%$ of GATA3 positive cases were also ER positive.

Similarly, $68.7 \%$ of PR positive cases showed GATA3 positivity, while only $46.7 \%$ of PR negative cases exhibited GATA3 positivity. PR status and GATA3 were found to have a significant association (Pearson $\chi^{2}$ test, $P=0.009$ ).

$60 \%$ of HER $2 /$ neu (score 0 and 1$)$ negative cases $(66.7 \%$ of the patient cohort) were GATA3 positive. In contrast, only $20 \%$ of HER $2 /$ neu (score 3 ) positive cases were GATA3 positive .This denotes statistically significant inverse association between GATA3 and HER2/neu (Pearson $\chi^{2}$ test, $\left.P=0.038\right)$.

$54.5 \%$ of cases with $\mathrm{Ki}-67<14 \%$ were GATA3 positive as compared to $42.1 \%$ of cases with Ki- $67 \geq 14 \%$. However, this difference was statistically insignificant. (Pearson $\chi^{2}$ test, $\left.P=0.51\right)$.

There was a strong association between GATA3 positive cases and grade 1 and 2 tumours (Pearson $\chi^{2}$ test, $P=$ 0.012 ). $100 \%$ of grade 1 tumours, $60 \%$ of grade 2 tumours and only $16.7 \%$ of grade 3 tumours exhibited GATA3 expression

The mean age in GATA3 positive cases was 48 years versus 54 years in GATA3 negative cases. But no significant association was found between GATA3 positivity and age at diagnosis (Mann-Whitney test, $P=0.224$ ).

GATA3 expression was not significantly associated with laterality of breast (Pearson $\chi^{2}$ test, $P=0.654$ ), histological type (Pearson $\chi^{2}$ test, $P=0.276$ ), tumour size (Linear by Linear Association, $P=0.639$ ), lymph node status (Linear by Linear Association, $P=0.453)$.

Correlation between clinicopathological variables with GATA3 expression are summarised in Table 2.

Table 1: Immunohistochemical labelling of GATA3 in cases of invasive breast carcinoma $(n=30)$.

\begin{tabular}{|c|c|c|c|c|c|c|c|}
\hline \multirow[t]{2}{*}{ Tumor type } & \multirow[t]{2}{*}{ n } & \multicolumn{5}{|c|}{ GATA3 } & \multirow[t]{2}{*}{ Total Positive } \\
\hline & & 0 & $1+$ & $2+$ & $3+$ & 4+ & \\
\hline Luminal A & $9(30 \%)$ & 4 & 0 & 1 & 2 & 2 & $5 / 9(55.56 \%)$ \\
\hline Luminal B & $10(33.4 \%)$ & 3 & 1 & 1 & 3 & 2 & $7 / 10(70 \%)$ \\
\hline Her 2 Neu enriched & $6(20 \%)$ & 5 & 0 & 1 & 0 & 0 & $1 / 6(16.66 \%)$ \\
\hline Triple Negative & $5(16.6 \%)$ & 4 & 0 & 1 & 0 & 0 & $1 / 5(20 \%)$ \\
\hline Total & 30 & 16 & & & & & $14 / 30(46.67 \%)$ \\
\hline
\end{tabular}

Table 2: Correlation between clinicopathological parameters and GATA3 expression.

\begin{tabular}{|l|l|l|l|l|}
\hline Parameter & $\mathbf{n}(\%)$ & GATA3+(\%) & GATA3- (\%) & p value \\
\hline Age & & & & \\
\hline $\mathbf{5 1}$ & $18(60)$ & $10(55.6)$ & $8(44.4)$ & 0.231 \\
\hline $\mathbf{5 1}$ & $12(40)$ & $4(33.3)$ & $8(66.7)$ & \\
\hline
\end{tabular}




\begin{tabular}{|c|c|c|c|c|}
\hline Parameter & $n(\%)$ & GATA3+(\%) & GATA3- (\%) & $p$ value \\
\hline \multicolumn{5}{|l|}{ Laterality of Breast } \\
\hline Right Breast Cancer & $12(40)$ & $5(41.7)$ & $7(38.3)$ & \multirow[t]{2}{*}{0.654} \\
\hline Left Breast Cancer & $18(60)$ & $9(50)$ & $9(50)$ & \\
\hline \multicolumn{5}{|l|}{ Histological Grade } \\
\hline 1 & $3(10)$ & $3(100)$ & $0(0)$ & \multirow[t]{3}{*}{0.012} \\
\hline 2 & $15(50)$ & $9(60)$ & $6(40)$ & \\
\hline 3 & $12(40)$ & $2(16.7)$ & $10(83.3)$ & \\
\hline \multicolumn{5}{|l|}{ ER } \\
\hline Positive & $18(60)$ & $12(66.7)$ & $6(33.3)$ & \multirow[t]{2}{*}{0.007} \\
\hline Negative & $12(40)$ & $2(16.7)$ & $10(83.3)$ & \\
\hline \multicolumn{5}{|l|}{ PR } \\
\hline Positive & $16(53.3)$ & $11(68.7)$ & $5(31.2)$ & \multirow[t]{2}{*}{0.009} \\
\hline Negative & $14(46.7)$ & $3(21.4)$ & $11(78.6)$ & \\
\hline \multicolumn{5}{|l|}{ HER2/neu } \\
\hline Positive & $10(33.3)$ & $2(20)$ & $8(80)$ & \multirow[t]{2}{*}{0.038} \\
\hline Negative & $20(66.7)$ & $12(60)$ & $8(40)$ & \\
\hline \multicolumn{5}{|l|}{ Ki 67} \\
\hline$<14 \%$ & $11(36.7)$ & $6(54.5)$ & $5(45.5)$ & \multirow[t]{2}{*}{0.51} \\
\hline$\geq 14 \%$ & $19(63.3)$ & $8(42.1)$ & $11(57.9)$ & \\
\hline \multicolumn{5}{|l|}{ Histological Type } \\
\hline IDC & $29(96.7)$ & $13(44.8)$ & $16(55.2)$ & \multirow[t]{2}{*}{0.276} \\
\hline Mucinous Ca & $1(2.3)$ & $1(100)$ & 0 & \\
\hline \multicolumn{5}{|l|}{ T Category } \\
\hline $\mathrm{T} 1$ & 0 & 0 & 0 & \multirow[t]{4}{*}{$0.639^{a}$} \\
\hline T2 & $4(13.3)$ & $1(25)$ & $3(75)$ & \\
\hline T3 & $5(16.7)$ & $1(20)$ & $4(80)$ & \\
\hline T4 & $1(3.3)$ & 0 & $1(100)$ & \\
\hline Not assessed & $20(66.7)$ & & & \\
\hline \multicolumn{5}{|l|}{ N Category } \\
\hline No & $4(13.3)$ & $1(25)$ & $3(75)$ & \multirow[t]{4}{*}{$0.453^{a}$} \\
\hline N1 & $3(10)$ & $1(33.3)$ & $2(66.7)$ & \\
\hline N2 & $2(6.7)$ & 0 & $2(100)$ & \\
\hline N3 & $1(3.3)$ & 0 & $1(100)$ & \\
\hline Not assessed & $20(66.7)$ & & & \\
\hline
\end{tabular}

ER: Estrogen Receptor, PR: Progesterone Receptor, Her2 neu: Human Epidermal Growth Factor Receptor. Pearson's $\chi 2$ test was used. P values $<0.05$ are shown in bold. ${ }^{a}$ Linear by linear association 


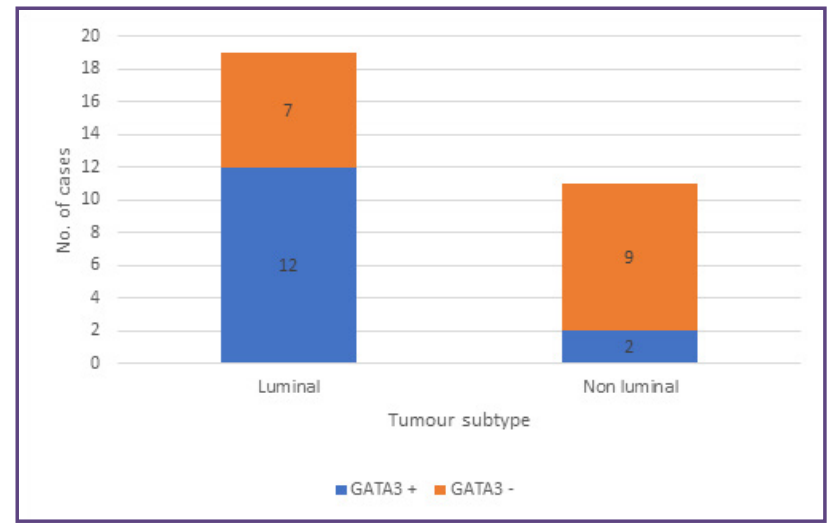

Fig. 1: GATA3 status according to tumour subtype among cases of invasive breast carcinoma $(n=30)$.

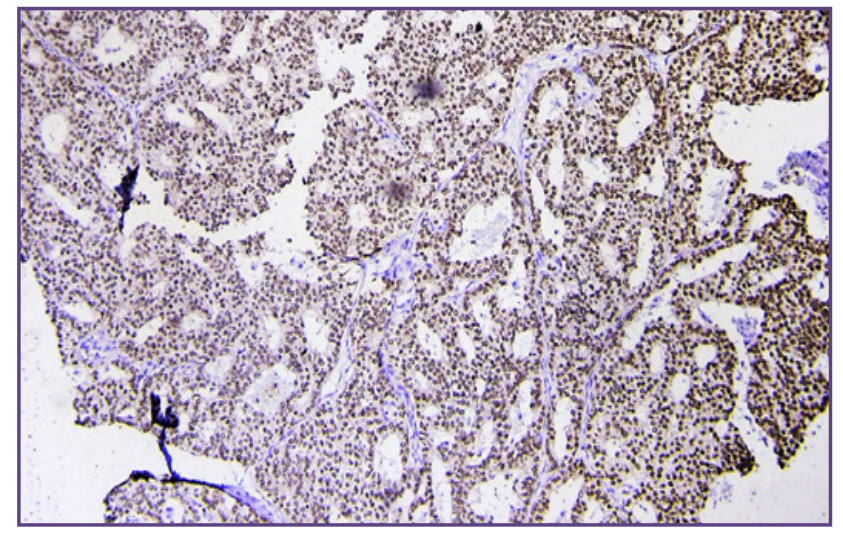

Fig. 2: GATA3 staining in invasive carcinoma breast (Nuclear;3+)(IHC;10X).

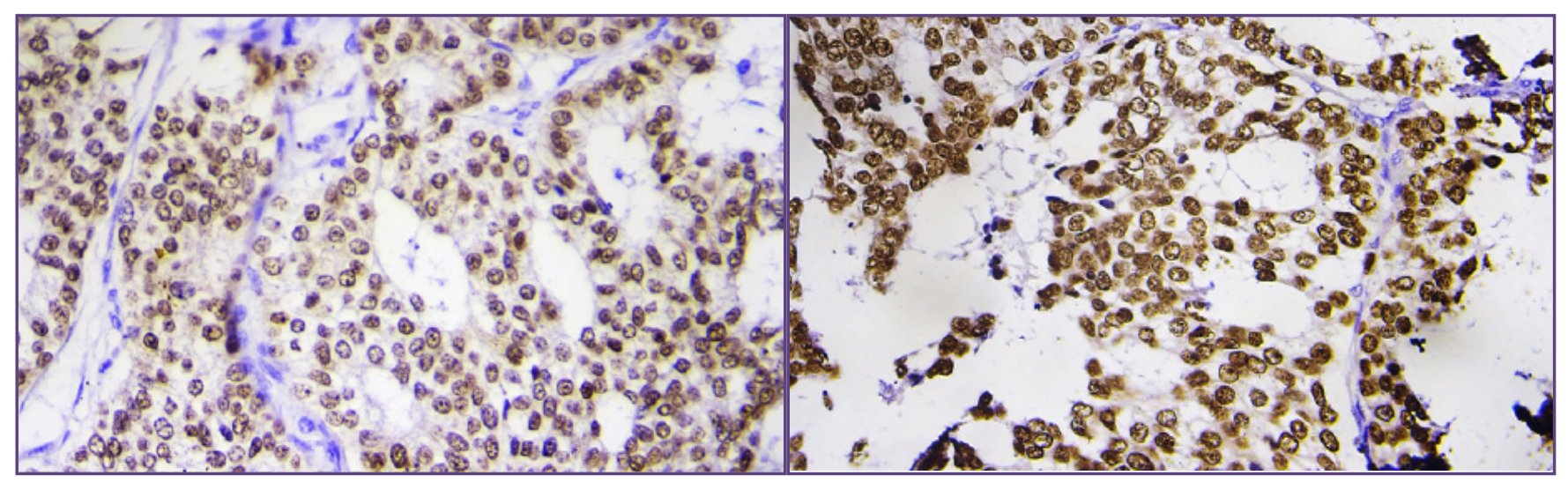

Fig. 3 A\&B: GATA3 staining in invasive carcinoma breast (Nuclear;3+)(IHC;40X).

\section{Discussion}

GATA3, a highly conserved and essential transcription factor, plays an integral role in cell fate specification. It is an important regulator of the development and commitment of luminal epithelial cells in the mammary gland. ${ }^{[3]}$ Since GATA3 promotes and specifies luminal cell identity in the mammary gland, it is involved in the pathogenesis of the luminal subtype in breast cancer too. GATA3 and ER proteins as components of the luminal transcriptional program may dictate the phenotype of hormonal-dependent breast cancer. ${ }^{[20]}$

The aim of this study was to determine whether expression of GATA3 correlated with pathological prognostic parameters in breast cancer. Previous works have highlighted the prognostic and predictive value of expression of GATA3 in breast carcinoma and shown using immunohistochemistry that it is closely correlated with ER $\alpha$ expression. ${ }^{[6-8,10,20]}$ In the present study too, the expression of GATA3 was directly associated with ER, PR and the luminal subtype. But some researches suggest the relationship between GATA3 and the ER stimulated pathways is not direct and may be bridged by the forkhead family transcription factor, FOXA1. ${ }^{[3,20]}$
Despite the strong association between GATA3 and ER expression in breast carcinomas, we found that there was a group of ER positive tumours expressing low GATA3. This may indicate that GATA3 levels may have prognostic value in patients with ER positive tumours.

GATA3 expression was inversely correlated with HER2/ neu overexpression ${ }^{[7,12]}$ and histological grade ${ }^{[7,8,10-12,14,19]}$ in our cohort as well as in others. Our data demonstrated that the highest GATA3 levels are seen in the welldifferentiated/ER positive/luminal breast cancers and its expression is inversely correlated with histologic grade/ tumour differentiation. This is in contrast to studies by McCleskey et al. ${ }^{[11]}$, Cimino-Mathews et al. ${ }^{[15]}$ and Byrne et al. ${ }^{[16]}$, who observed significant GATA3 expression in non-luminal breast carcinomas (including triple negative) as well.

In our patient cohort, GATA3 immunopositivity was observed in $46.7 \%$ cases. The proportion of GATA3 expression varies widely among different studies. This discrepancy could be explained by geographic variability, sample size, choice of the antibody used, differences in 
immunohistochemical protocols and different scoring methods. Therefore, multicentric studies are required to confirm GATA3 expression.

The current study found that common clinicopathologic parameters including patient age at diagnosis, laterality of breast, histological type, tumour size, nodal status were all statistically insignificant. Similar to our findings, Voduc et al. concluded no significant association with lymph node status. But they did find a linear association between age at diagnosis/tumour size and GATA3 expression although the actual difference between means was small. ${ }^{[12]}$ Some studies did show a statistically significant association between tumour size ${ }^{[7,8,12]}$, lymph node status ${ }^{[7]}$ and even metastasis. ${ }^{[1,16,17]}$ pTNM data of only 10 of the 30 cases included in this study was available, which may have contributed to the incongruity of our results with those of other studies.

Based on our results, we can consider GATA3 as a transcription factor that regulates the ER pathway which may be important to the luminal phenotype of breast cancer. This is an independent validation of many similar studies and opens up avenues to gauge the prognostic value of GATA3 by assessing relapse, disease free survival and response to therapeutic strategies.

\section{Conclusion}

Based on immunohistochemical evaluation of GATA3 in patients of invasive breast carcinoma, we found GATA3 to be a breast cancer marker almost exclusively expressed among ER positive and PR positive tumours. Similar to the ER, it is associated with favourable prognostic features like lower tumour grade and the absence of HER2 overexpression.

Presently, prediction of tumour progression and selection of individualised therapies based on molecular targets are the major obstacles in oncology. Nowadays, ER expression alone is being used to assess patient prognosis and guide hormone-based treatment in breast cancer. However, not all ER positive carcinomas react similarly to such therapies or show comparable prognosis. Therefore, there is a need to refine the molecular classification of tumours in order to better predict their clinical course and the patient's response to current therapies. GATA3 might be useful in this respect because, as stated earlier, GATA3 expression was found to be low in some ER positive cases.

The expression of GATA3 as an ER associated gene provides a chance to identify patients that will have a good prognosis. Although our results are promising, they need to be validated in relationship to outcome in a larger prospective cohort. But assessment of the value of GATA3 in the therapeutic response setting and in metastatic cases was beyond the scope of this study. Further investigation into GATA3 levels in multiple separate and independent patient populations will greatly strengthens its potential utility as a prognostic indicator and will be crucial to our understanding of breast cancer dissemination and recurrence early in the progression of breast cancer. It may be significant as predictor of pathological complete remission (pCR). This would help in better patient management and enable us to reliably assess the prognosis of patients diagnosed with breast cancer allowing accurate treatment modalities, efficient adjustment and monitoring of neoadjuvant/chemotherapy/hormonal therapy and decrease in treatment failure, risks of therapies, side effects and remission.

Nonetheless, the present study already offers some headway in the overview of breast cancer; GATA3 was significantly associated with ER positive tumours and low tumour grade. But this marker should not be used in isolation. Careful evaluation of the cytomorphology, clinical history, and radiologic findings, as well as the use of a panel of immunomarkers are important.

\section{Acknowledgements}

I am thankful for the support received from ICMR under the STS program. I am grateful to Dr Rashmi Arora, HOD, Pathology, Safdarjung Hospital for giving permission to conduct the study. Special thanks to Dr Sakshi Batra and Ms Anu for their assistance throughout the study.

\section{Funding}

None

\section{Competing Interests}

None

\section{Reference}

1. Bray F, Ferlay J, Soerjomataram I, Siegel R, Torre L, Jemal A. Global cancer statistics 2018: GLOBOCAN estimates of incidence and mortality worldwide for 36 cancers in 185 countries. CA Cancer J Clin. 2018;68(6):394-424.

2. Malvia S, Bagadi S, Dubey U, Saxena S. Epidemiology of breast cancer in Indian women. Asia Pac J Clin Oncol. 2017;13(4):289-95.

3. Kouros-Mehr H, Slorach E, Sternlicht M, Werb Z. GATA-3 maintains the differentiation of the luminal cell fate in the mammary gland. Cell. 2006;127(5):1041-55.

4. Kouros-Mehr H, Bechis S, Slorach E, Littlepage L, Egeblad M, Ewald A et al. GATA-3 links tumor differentiation and dissemination in a luminal breast cancer model. Cancer Cell. 2008;13(2):141-52.

5. Liu H, Shi J, Wilkerson ML, Lin F. Immunohistochemical evaluation of GATA3 expression in tumors and normal 
tissues: a useful immunomarker for breast and urothelial carcinomas. Am J Clin Pathol. 2012;138 (1):57-64.

6. Hoch RV, Thompson DA, Baker RJ, Weigel RJ. GATA-3 is expressed in association with estrogen receptor in breast cancer. Int J Cancer. 1999 Apr 20;84(2):122-8.

7. Mehra R, Varambally S, Ding L, Shen R, Sabel M, Ghosh D et al. Identification of GATA3 as a breast cancer prognostic marker by global gene expression meta-analysis. Cancer Res. 2005;65(24):11259-64.

8. Yoon N, Maresh E, Shen D, Elshimali Y, Apple S, Horvath $\mathrm{S}$ et al. Higher levels of GATA3 predict better survival in women with breast cancer. Hum Pathol. 2010;41(12):17941801.

9. Fararjeh AS, Tu SH, Chen LC, Liu YR, Lin YK, Chang HL, et al. The impact of the effectiveness of GATA3 as a prognostic factor in breast cancer. Hum Pathol. 2018 10;80:219-30.

10. Cakir A, Isik Gonul I, Ekinci O, Cetin B, Benekli M, Uluoglu O. GATA3 expression and its relationship with clinicopathological parameters in invasive breast carcinomas. Pathol Res Pract. 2017 Mar;213(3):227-34.

11. McCleskey B, Penedo T, Zhang K, Hameed O, Siegal G, Wei S. GATA3 Expression in advanced breast cancer: prognostic value and organ-specific relapse. Am J Clin Pathol. 2015;144(5):756-63.

12. Voduc D, Cheang M, Nielsen T. GATA-3 expression in breast cancer has a strong association with estrogen receptor but lacks independent prognostic value. Cancer Epidemiol Biomarkers Prev. 2008;17(2):365-73.

13. Shaoxian T, Baohua Y, Xiaoli X, Yufan C, Xiaoyu T, Hongfen L, et al. Characterisation of GATA3 expression in invasive breast cancer: differences in histological subtypes and immunohistochemically defined molecular subtypes. J Clin Pathol. 2017 Nov;70(11):926-34.

14. Demir H, Turna H, Can G, Ilvan S. Clinicopathologic and prognostic evaluation of invasive breast carcinoma molecular subtypes and GATA3 expression. J BUON. 2010;15(4):774-82.

15. Cimino-Mathews A, Subhawong A, Illei P, Sharma R, Halushka M, Vang R et al. GATA3 expression in breast carcinoma: utility in triple-negative, sarcomatoid, and metastatic carcinomas. Hum Pathol. 2013;44(7):1341-49.

16. Byrne D, Deb S, Takano E, Fox S. GATA3 expression in triplenegative breast cancers. Histopathology. 2017;71(1):63-71.

17. Braxton DR, Cohen C, Siddiqui MT. Utility of GATA3 immunohistochemistry for diagnosis of metastatic breast carcinoma in cytology specimens. Diagn Cytopathol. 2015 Apr;43(4):271-7.

18. Ciocca V, Daskalakis C, Ciocca R, Ruiz-Orrico A, Palazzo $\mathrm{J}$. The significance of GATA3 expression in breast cancer: a 10-year follow-up study. Hum Pathol. 2009;40(4):489-95.

19. Gulbahce HE, Sweeney C, Surowiecka M, Knapp D, Varghese L, Blair CK. Significance of GATA-3 expression in outcomes of patients with breast cancer who received systemic chemotherapy and/or hormonal therapy and clinicopathologic features of GATA-3-positive tumors. Hum Pathol. 2013 Nov;44(11):2427-31.

20. Eeckhoute J, Keeton E, Lupien M, Krum S, Carroll J, Brown M. Positive cross-regulatory loop ties GATA-3 to estrogen receptor $\alpha$ expression in breast cancer. Cancer Res. 2007;67(13):6477-83.

21. Layton C, Bancroft JD. The hematoxylin and eosin. In: Suvarna SK, Layton C, Bancroft JD, editors. Theory and practice of histological techniques. 7th ed. Philadelphia: Elsevier; 2013. pp.179-220.

22. Collins LC. Breast. In: Rosai and Ackerman, editors. Surgical Pathology. 11th ed. Philadelphia: Elsevier; 2017. pp.1434-527.

23. Singletary SE, Allred C, Ashley P, Berry D, Bland KI, Borgen PI et al. Revision of the American Joint Committee on Cancer staging system for breast cancer. J Clin Oncol 2002;20:3628-36.

*Corresponding author:

Tanvi Suri, B-3/8 Safdarjung Enclave, New Delhi, Delhi-110029

Phone: +919540443399

Email: tanvisuri99@gmail.com

Financial or other Competing Interests: None.

$\begin{array}{ll}\text { Date of Submission } & : \text { 26/12/2020 } \\ \text { Date of Final Revision } & : 09 / 02 / 2021 \\ \text { Date of Acceptance } & : 11 / 02 / 2021 \\ \text { Date of Publication } & : \text { 28/02/2021 }\end{array}$

